\title{
ON A VARIATIONAL PRINCIPLE FOR THE NAVIER-STOKES EQUATION
}

\author{
DIOGO AGUIAR GOMES
}

\begin{abstract}
In this paper we study the variational principle for the Navier-Stokes equation described in Gom05, and clarify the role of boundary conditions. We show that in certain special cases this variational principle gives rise to new models for fluid equations.
\end{abstract}

\section{Contents}

1. Introduction 1

2. A non-probabilistic variational principle 3

3. Conservation of Energy 5

$\begin{array}{lll}\text { A. Navier-Stokes equation in magnetization variables } & 6\end{array}$

$\begin{array}{ll}\text { References } & 7\end{array}$

Supported in part by FCT/POCTI/FEDER and POCI/MAT/55745/2004

\author{
Departamento de Matemática, \\ Instituto Superior Técnico \\ Av. Rovisco Pais \\ 1049-001 Lisboa, Portugal \\ email:dgomes@math.ist.utl.pt
}

\section{INTRODUCTION}

Several authors, for instance Rap01, Rap00, [Bus99], [BFR], CI05] have studied representation formulas for solutions of the Navier-Stokes 
equation using probabilistic methods, and, in fact, the idea of using random maps instead of deterministic ones can be traced back to Chorin Cho73] and Peskin Pes85. In a completely different setting, Arnold Arn66, and Ebin and Marsden [EM70, proved a variational principle for the solutions of Euler equation. It is of course natural to ask whether there is a probabilistic analog for the Navier-Stokes equation. In fact, there is at least one, non-probabilistic variational principle for the Navier-Stokes equation[]. However, recently stochastic variational principles for the Navier-Stokes equation have drawn attention and at least three different ones have been proposed by several authors: [IF79], Cru05, and Gom05]. This paper builds upon the last variational principle and is a contribuition to its study. This variational principle can be stated as follows:

Theorem 1. Suppose $\left(u, \phi^{\omega}\right)$ is a critical point, $u$ smooth in spacetime, and $\phi$ smooth in space and $C^{1}$ in time, of

$$
\hat{S}=\int_{0}^{T} \int_{\mathbb{R}^{n}} \frac{|u|^{2}}{2}+E \int_{\mathbb{R}^{n}} \Theta\left(\phi^{\omega}(x, T)\right)
$$

under the constraint $\operatorname{div} u=0$, and

$$
\frac{\partial \phi^{\omega}}{\partial t}=u \circ B_{t} \circ \phi^{\omega}
$$

and $\phi^{\omega}(x, 0)=x$. Assume further that the function $\Pi^{\omega}$ which satisfies the differential equation

$$
\frac{\partial \Pi_{i}^{\omega}}{\partial t}+\Pi_{j}^{\omega} D_{i} u_{j} \circ B_{t} \circ \phi^{\omega}=0,
$$

with the terminal condition

$$
\Pi^{\omega}(x, T)=D \Theta\left(\phi^{\omega}(x, T)\right),
$$

is, at $t=0$, non-random. Then $u$ is a solution to the Navier-Stokes equation.

One can relate some of the objects that arise in this last theorem to the works by P. Constantin in [Con01a, Con01b, Con03]. In fact, consider a minimizer $\left(u, \phi^{\omega}\right)$ as in the previous theorem, and define

$$
A=E\left[\left(\phi^{\omega}\right)^{-1} \circ B_{t}^{-1}\right] .
$$


ON A VARIATIONAL PRINCIPLE FOR THE NAVIER-STOKES EQUATION 3

The next proposition shows that the vector $A$ satisfies exactly the advection-diffusion equation as in Con01b].

Proposition 1. The vector A satisfies

$$
\frac{\partial A}{\partial t}+(u \cdot \nabla) A-\frac{1}{2} \Delta A=0
$$

with $A(x, 0)=x$. Note that this does not depend on $u$ being a solution to the Navier-Stokes equation.

In this paper we study a variation of theorem 1, with a special terminal cost which enable to remove the probability from the variational principle. However, the vector $\Pi$ will not be in general progressively measurable, hence the critical points may not be solutions to the Navier-Stokes equation. This gives rise to a new model equation for incompressible viscous fluids and we study some of its properties.

\section{A NON-PROBABILISTIC VARIATIONAL PRINCIPLE}

Consider the case of a terminal cost which depends only on the active transport vector $A$, and given deterministic data. For instance

$$
\frac{1}{2} \int_{\mathbb{R}^{n}}|A(x, T)-\psi(x, T)|^{2} .
$$

Then, we may replace the variable $\phi^{\omega}$ by the variable $A$, and so we may consider the problem

$$
\min \int_{0}^{T} \int_{\mathbb{R}^{n}} \frac{|u|^{2}}{2}+\frac{1}{2} \int_{\mathbb{R}^{n}}|A(x, T)-\psi(x)|^{2},
$$

under the constraints

$$
\operatorname{div} u=0, \quad \partial_{t} A+(u \cdot \nabla) A-\frac{1}{2} \Delta A=0 .
$$

Theorem 2. Let $(u, A)$ be a critical point of the functional (3) under the constraints (4). Let $\zeta$ be the solution of

$$
\partial_{t} \zeta_{i}-\partial_{j}\left(\zeta_{i} u_{j}\right)+\frac{1}{2} \Delta \zeta_{i}=0,
$$

with the terminal condition

$$
\zeta(x, T)=A(x, T)-\psi(x) .
$$


Then $u=\mathbb{P} m$, where $m_{i}=\zeta_{j} \partial_{i} A_{j}$ satisfies the modified Navier-Stokes equation in magnetization form:

$$
\partial_{t} m_{i}+u_{j} D_{j} m_{i}+m_{j} D_{i} u_{j}=\frac{1}{2} \Delta m_{i}-\partial_{k}\left(\left(\partial_{k} \zeta_{j}\right)\left(\partial_{i} A_{j}\right)\right)
$$

REMARK. (6) should be compared with the corresponding equation for the Navier-Stokes equation, equation (8) in the appendix.

Proof. We will use the variable $\zeta$ as a Lagrange multiplier to make the computation of the Euler-Lagrange equation easier. Thus consider the equivalent problem of minimizing

$$
\int_{0}^{T} \int_{\mathbb{R}^{n}} \frac{|u|^{2}}{2}+\left\langle\zeta, \partial_{t} A+(u \cdot \nabla) A-\frac{1}{2} \Delta A\right\rangle+\frac{1}{2} \int_{\mathbb{R}^{n}}|A(x, T)-\psi(x)|^{2}
$$

under the same constraints. By making variations $A+\epsilon \delta A$ and $u+\epsilon \delta u$ we obtain

$$
\int_{0}^{T} \int_{\mathbb{R}^{n}}\langle u, \delta u\rangle+\langle\zeta,(\delta u \cdot \nabla) A\rangle=0
$$

which implies $u=\mathbb{P} m$ with $m_{i}=\zeta_{j} \partial_{i} A_{j}$, and

$$
\begin{aligned}
\int_{0}^{T} \int_{\mathbb{R}^{n}}\left\langle\zeta, \partial_{t} \delta A+(u \cdot \nabla) \delta A\right. & \left.-\frac{1}{2} \Delta \delta A\right\rangle \\
& +\int_{\mathbb{R}^{n}}\langle A(x, T)-\psi(x), \delta A(x, T)\rangle=0,
\end{aligned}
$$

which shows that

$$
\zeta(x, T)=A(x, T)-\psi(x)
$$

after integration by parts (in space and time) and using the definion of $\zeta$.

Finally, we must show that $m$ satisfies $(6)$. To this end, observe that

$$
\begin{aligned}
\partial_{t}\left(\zeta_{j} \partial_{i} A_{j}\right)= & \partial_{t} \zeta_{j} \partial_{i} A_{j}+\zeta_{j} \partial_{i}\left(u_{k} \partial_{k} A_{j}+\frac{1}{2} \Delta A_{j}\right) \\
= & \partial_{k}\left(\zeta_{j} u_{k}\right) \partial_{i} A_{j}+\zeta_{j} \partial_{i}\left(u_{k} \partial_{k} A_{j}\right)+\frac{1}{2}\left(\zeta_{j} \partial_{i} \Delta A_{j}-\Delta \zeta_{j} \partial_{i} A_{j}\right) \\
= & u_{k} \partial_{k} m_{i}+m_{k} \partial_{i} u_{k} \\
& \quad+\frac{1}{2}\left(\zeta_{j} \partial_{i} \Delta A_{j}-\Delta \zeta_{j} \partial_{i} A_{j}\right)
\end{aligned}
$$


To finish the proof, we just have to observe that

$$
\begin{aligned}
\frac{1}{2}\left(\zeta_{j} \partial_{i} \Delta A_{j}-\Delta \zeta_{j} \partial_{i} A_{j}\right) & =\frac{1}{2} \Delta m_{i}-\left(\Delta \zeta_{j} \partial_{i} A_{j}+\partial_{k} \zeta_{j} \partial_{i} \partial_{k} A_{j}\right) \\
& =\frac{1}{2} \Delta m_{i}-\partial_{k}\left(\left(\partial_{k} \zeta_{j}\right)\left(\partial_{i} A_{j}\right)\right) .
\end{aligned}
$$

By applying the Leray projection to (6) we obtain that $u$ solves

$$
\partial_{t} u+u \cdot \nabla u=\frac{1}{2} \Delta u+\nabla p-\mathbb{P}\left(\partial_{k}\left(\left(\partial_{k} \zeta_{j}\right)\left(\partial_{i} A_{j}\right)\right)\right)
$$

Finally, we should observe that in the case of no-viscosity, the proof of this theorem is a very short and elementary proof of the well known result by Arnold [Arn66], and Ebin and Marsden [EM70], for the solutions of Euler's equation, as using the active vector $A$ which is the inverse of the flow map instead of the flow map simplifies a lot the computations.

\section{Conservation of Energy}

Whereas the original variational problem in Gom05] does involve some explicit time dependence through the Brownian motion, the modified variational principle we study in this problem does not involve time explicitly. Therefore, by Noether's theorem, there should be a conserved quantity, the energy, corresponding to this symmetry. This is exactly the content of the next theorem.

Theorem 3 (Energy conservation). The energy

$$
\int_{\mathbb{R}^{n}} \frac{|u|^{2}}{2}-\left\langle\partial_{t} \zeta, A\right\rangle
$$

a constant of motion for the modified Navier-Stokes equation (6).

Proof. Although this conservation law can be derived directly, it is quite instructive to do the proof following the steps of Noether's theorem. 
We have

$$
\begin{aligned}
& \int_{h}^{T+h} \int_{\mathbb{R}^{n}} \frac{|u(x, t-h)|^{2}}{2} \\
& +\left\langle\zeta(x, t-h), \partial_{t} A(x, t-h)+(u(x, t-h) \cdot \nabla) A(x, t-h)\right. \\
& \left.\quad-\frac{1}{2} \Delta A(x, t-h)\right\rangle
\end{aligned}
$$

is independent of $h$. Therefore, if we differentiate in $h$ at $h=0$ we obtain

$$
\begin{aligned}
\frac{|u(x, T)|^{2}}{2}-\frac{|u(x, 0)|^{2}}{2} & =\int_{0}^{T} \int_{\mathbb{R}^{n}}\left\langle u, \partial_{t} u\right\rangle \\
& +\left\langle\zeta, \partial_{t t}^{2} A+\partial_{t} u \cdot \nabla A+u \cdot \nabla \partial_{t} A-\frac{1}{2} \Delta \partial_{t} A\right\rangle .
\end{aligned}
$$

Now observe that

$$
\int_{0}^{T} \int_{\mathbb{R}^{n}}\left\langle u, \partial_{t} u\right\rangle+\left\langle\zeta, \partial_{t} u \cdot \nabla A\right\rangle=0
$$

Furthermore, integrating by parts, we have

$$
\begin{aligned}
& \int_{0}^{T} \int_{\mathbb{R}^{n}}\left\langle\zeta, \partial_{t t}^{2} A+u\right.\left.\cdot \nabla \partial_{t} A-\frac{1}{2} \Delta \partial_{t} A\right\rangle \\
&=\int_{\mathbb{R}^{n}}\left\langle\zeta(x, T), \partial_{t} A(x, T)\right\rangle-\left\langle\zeta(x, 0), \partial_{t} A(x, 0)\right\rangle .
\end{aligned}
$$

Since $\partial_{t} A=-u \cdot \nabla A-\frac{1}{2} \Delta A$ we conclude that

$$
\int_{\mathbb{R}^{n}} \frac{|u|^{2}}{2}-\left\langle\zeta, u \cdot \nabla A+\frac{1}{2} \Delta A\right\rangle
$$

is a constant of motion. Taking into account equation (5) we finish the proof.

\section{A. NAVier-Stokes EquATion in MAgnetization VARiables}

In this appendix we review the magnetization form for the NavierStokes equation in $\mathbb{R}^{n}$ for the velocity field $u(x, t)$ of an incompressible fluid:

$$
u_{t}+(u \cdot \nabla) u+\nabla p=\frac{1}{2} \Delta u \quad \operatorname{div} u=0,
$$

with initial condition $\left.u\right|_{t=0}=u^{0}$. The variable $p(x, t)$ is the pressure and is necessary to impose the incompressibility condition $\operatorname{div} u=0$. 
For our purposes in this paper, it is convenient to rewrite (7) in new variables, the magnetization variables. These have been used to study the Euler equation by several authors, namely Buttke [But93], Oseledets [Ose89], Russo and Smereka [RS99], among others. We will follow Chorin [Cho94] in the summary of results we present next.

The magnetization variable $m$ is obtained by adding to the velocity field $u$ a gradient

$$
u=m+\nabla k \text {. }
$$

The scalar function $k(x, t)$ is arbitrary at $t=0$ and its evolution is chosen conveniently.

This transformation is a change of gauge, of which there are several possible choices, as discussed in [RS99]. Clearly, from $m$ one can compute $u$ by using the Leray projection on the divergence free vector fields:

$$
u=\mathbb{P} m \text {. }
$$

With an appropriate choice for $k$, the equation for the evolution of $m$ is

$$
\partial_{t} m_{i}+u_{j} D_{j} m_{i}+m_{j} D_{i} u_{j}=\frac{1}{2} \Delta m_{i}
$$

A main difference from (7) is that equation (8) does not involve pressure, nor $\operatorname{div} m=0$. Furthermore, to any solution of (8) with $u=\mathbb{P} m$, corresponds a solution $u$ to (7). In the other direction, to any solution of (7) and initial value of $k$ there exists a solution of (8) such that $u=\mathbb{P} m$ for all times.

\section{REFERENCES}

[Arn66] V. Arnold. Sur la géométrie différentielle des groupes de Lie de dimension infinie et ses applications à l'hydrodynamique des fluides parfaits. Ann. Inst. Fourier (Grenoble), 16(fasc. 1):319-361, 1966.

[BFR] Barbara Busnello, Franco Flandoli, and Marco Romito. A probabilistic representation for the vorticity of a 3-dimensional viscous fluid and for general systems of parabolic equations.

[Bus99] Barbara Busnello. A probabilistic approach to the two-dimensional Navier-Stokes equations. Ann. Probab., 27(4):1750-1780, 1999. 
[But93] Tomas F. Buttke. Velicity methods: Lagrangian numerical methods which preserve the Hamiltonian structure of incompressible fluid flow. In Vortex flows and related numerical methods (Grenoble, 1992), volume 395 of NATO Adv. Sci. Inst. Ser. C Math. Phys. Sci., pages 39-57. Kluwer Acad. Publ., Dordrecht, 1993.

[Cho73] Alexandre Joel Chorin. Numerical study of slightly viscous flow. J. Fluid Mech., 57(4):785-796, 1973.

[Cho94] Alexandre J. Chorin. Vorticity and turbulence, volume 103 of Applied Mathematical Sciences. Springer-Verlag, New York, 1994.

[CI05] P. Constantin and G. Iyer. A stochastic lagrangian representation of 3dimensional incompressible navier-stokes equations. 2005.

[Con01a] Peter Constantin. An Eulerian-Lagrangian approach for incompressible fluids: local theory. J. Amer. Math. Soc., 14(2):263-278 (electronic), 2001.

[Con01b] Peter Constantin. An Eulerian-Lagrangian approach to the Navier-Stokes equations. Comm. Math. Phys., 216(3):663-686, 2001.

[Con03] Peter Constantin. Near identity transformations for the Navier-Stokes equations. In Handbook of mathematical fluid dynamics, Vol. II, pages 117-141. North-Holland, Amsterdam, 2003.

[Cru05] Ana Bela Cruzeiro. preprint. 2005.

[EM70] David G. Ebin and Jerrold Marsden. Groups of diffeomorphisms and the notion of an incompressible fluid. Ann. of Math. (2), 92:102-163, 1970.

[Gom05] Diogo Aguiar Gomes. A variational formulation for the Navier-Stokes equation. Comm. Math. Phys., 257(1):227-234, 2005.

[IF79] Atsushi Inoue and Tadahisa Funaki. On a new derivation of the NavierStokes equation. Comm. Math. Phys., 65(1):83-90, 1979.

[Ose89] V. I. Oseledets. A new form of writing out the Navier-Stokes equation. Hamiltonian formalism. Uspekhi Mat. Nauk, 44(3(267)):169-170, 1989.

[Pes85] Charles S. Peskin. A random-walk interpretation of the incompressible Navier-Stokes equations. Comm. Pure Appl. Math., 38(6):845-852, 1985.

[Rap00] D. L. Rapoport. Stochastic differential geometry and the random integration of the Navier-Stokes equations and the kinematic dynamo problem on smooth compact manifolds and Euclidean space. Hadronic J., 23(6):637-675, 2000.

[Rap01] D. L. Rapoport. Random representations of viscous fluids and the passive magnetic fields transported on them. Discrete Contin. Dynam. Systems, (Added Volume):327-336, 2001. Dynamical systems and differential equations (Kennesaw, GA, 2000). 
ON A VARIATIONAL PRINCIPLE FOR THE NAVIER-STOKES EQUATION 9

[RS99] Giovanni Russo and Peter Smereka. Impulse formulation of the Euler equations: general properties and numerical methods. J. Fluid Mech., 391:189-209, 1999. 